\title{
Synthesis and Physical Properties of Regioregular Poly(3-alkoxy-4-methylthiophene)s
}

\author{
Kazumasa TANAKA, Takashi MiHARA, and Naoyuki KOIDE ${ }^{\dagger}$ \\ Department of Chemistry, Faculty of Science, Tokyo University of Science, \\ 1-3 Kagurazaka, Shinjuku-ku, Tokyo 162-8601, Japan
}

(Received March 9, 2004; Accepted May 17, 2004; Published August 15, 2004)

\begin{abstract}
We synthesized poly(3-alkoxy-4-methylthiophene)s (PMThO) by conventional oxidative polymerization using iron trichloride to investigate the effect of length of the alkoxy chain introduced into the 3-position of the thiophene ring on the physical properties. Regioregular PMThO derivatives were obtained and the regioregularity of PMThO derivatives was confirmed by ${ }^{1} \mathrm{H}$ NMR and ${ }^{13} \mathrm{C}$ NMR measurements. In the UV-vis spectra of PMThO derivatives, vibronic peaks characteristic of the regioregular polythiophene derivatives were observed around $540 \mathrm{~nm}$, and new peaks of $275 \mathrm{~nm}$ and $400 \mathrm{~nm}$ assigned to the PMThO derivatives with non-planar conformation for the polymer backbone appeared with increasing temperature. The band gap energy of PMThO derivatives was about $1.9 \mathrm{eV}$ that was lower than that of conventional polythiophenes. The electrical conductivity of doped PMThO derivatives bearing longer alkoxy chains was about $10^{0} \mathrm{~S} \mathrm{~cm}^{-1}$. [DOI 10.1295/polymj.36.628]

KEY WORDS $\pi$-Conjugated Polymer / Chemical Oxidative Polymerization / Regioregular Polythiophene /
\end{abstract}

$\pi$-Conjugated polymers are investigated intensively due to their potential applications in electrical and optical devices. ${ }^{1}$ The $\pi$-conjugated polymers generally have rigid-rod nature based on the $\pi$-conjugation in the polymer backbone. The rigid-rod $\pi$-conjugated polymers had trouble to process because of their inherent insolubility in common organic solvents and infusibility. The introduction of a long and flexible chain into the side chain of the rigid-rod polymer backbone would result in a soluble and fusible rigidrod $\pi$-conjugated polymer. ${ }^{2-7}$

The conductivity of rigid rod $\pi$-conjugated polymers is changed remarkably by the regioregularity and the orientation of the polymer backbone. By substituting a long and flexible chain at the 3-position of the thiophene ring, one can decrease the interaction between polymer backbones and then achieves high solubility and fusibility.

The alkylthiophene monomers that are not centrosymmetric are used to obtain the rigid-rod poly(3alkylthiophene)s (PATs). The alkylthiophene monomers can couple in three different manners: head-totail (HT), head-to-head (HH) and tail-to-tail (TT). The three different manners lead to the four types of triads such as HT-HT, HT-HH, TT-HT and TT-HH in the PATs. ${ }^{8}$

Regioregular PATs have almost $100 \%$ of HT couplings in the polymer backbone. The $\pi$-conjugated length is expanded in the polymer backbone of regioregular polythiophene where the thiophene rings adjoin each other in the HT-HT linkage manner.
The regioregularity of the PATs played an important role for the enhancement of the electrical conductivity. Chen et al. reported that the regioregular PATs exhibited a larger conductivity than the regiorandom PATs. ${ }^{9}$ The regioregular PATs doped with $\mathrm{I}_{2}$ vapor heavily had conductivity around $10^{3} \mathrm{~S} \mathrm{~cm}^{-1}$, while the regiorandom PATs had conductivity less than $10^{1} \mathrm{~S} \mathrm{~cm}^{-1}$.

Generally regioregular PATs were synthesized using Rieke zinc or nickel catalyzed cross-coupling. ${ }^{9-13}$ However, the Rieke zinc or nickel catalyzed crosscoupling would need a special technique to handle for preparing the regioregular polythiophene (PTh) derivatives, while oxidative polymerization using iron trichloride of thiophene derivative is very simple method to get polythiophene derivative. On the other hand, polymerization of 3,4-disubstituted thiophenes is a useful way to prevent undesirable $\alpha, \beta$ coupling of the thiophene ring. No substituent at 4-position of the thiophene ring would lead to irregular coupling or crosslinking site, then the polythiophene derivatives obtained are insufficient to be completely soluble and to have high regioregular polymer backbone. Therefore, the polymerization of 3,4-disubstituted thiophene would have a great potential for giving structurally regular PTh derivatives. Leclerc et al. reported that regioregular 3,4-disubstituted PTh derivatives were obtained by chemical oxidation of the relative monomers by iron trichloride. ${ }^{14-19}$ This method is very easy way to obtain regioregular PTh derivatives compared to the synthetic methods using Rieke zinc

†To whom correspondence should be addressed (Tel: +81-3-5228-8248, Fax: +81-3-3235-2214, E-mail: nkoide@ch.kagu.sut.ac.jp). 
or nickel catalyzed cross-coupling. Leclerc et al. investigated intensively the thermochromism of regioregular 3,4-disubstituted polythiophenes with relatively short alkoxy chain.

In our preliminary experiments, poly(3-butoxy-4methylthiophene) (PMThO4) had more dominant effect on the regioregularity and on the electrical conductivity than poly(3-butyl-4-methylthiophene) (PMTh4). ${ }^{20}$ PMThO4 had a highly regioregular structure, while PMTh4 had a regiorandom structure. These results were supported by UV-vis spectroscopy and ${ }^{13} \mathrm{C}$ NMR measurements. The electrical conductivity of PMThO4 doped with iodine vapor was higher than that of PMTh4. Therefore, we considered that with regard to the electrical conductivity, the polythiophene derivative with alkoxy group would be superior to that with alkyl group.

Groenendaal et al. reported that the electrical conductivity of alkylated poly(3,4-ethylenedioxythiophene)s increased with increasing alkyl chain length $\left(\mathrm{C}_{6} \mathrm{H}_{13}, \mathrm{C}_{10} \mathrm{H}_{21}, \mathrm{C}_{14} \mathrm{H}_{29}\right) .{ }^{21} \mathrm{~A}$ high electrical conductivity of $850 \mathrm{~S} \mathrm{~cm}^{-1}$ was found for the tetradecyl-substituted poly(3,4-ethylenedioxythiophene) derivative. This result demonstrates that the long alkyl chain introduced into the side chain of polythiophenes does not always diminish the electrical conductivity.

On the basis of these two results, we, in this study, synthesized poly(3-alkoxy-4-methylthiophene) (PMThO) derivatives having a methyl group at the 4-position and a different length of alkoxy chain at the 3-position in the thiophene ring by the chemical oxidative polymerization catalyst, and investigated the relationship between the regioregularity and electrical conductivity, and optical properties of the PMThO derivatives.

\section{EXPERIMENTAL}

\section{Materials}

Synthesis of PMThO derivatives are summarized in Scheme 1 . The typical synthetic procedure of PMThO derivatives are described below.

\section{3-Hexyloxy-4-methylthiophene (MThO6)}

A solution of sodium methylate in NMP $(10 \mathrm{~mL})$ was heated to $100^{\circ} \mathrm{C}$ and then added $1.0 \mathrm{~g}$ of copper(I) bromide and $5.0 \mathrm{~g}$ of 3-bromo-4-methylthiophene. The reaction mixture was refluxed for $1 \mathrm{~h}$ under nitrogen atmosphere. The suspension was filtered and extracted with diethyl ether. The organic layer was washed with $\mathrm{NH}_{4} \mathrm{Cl}$ aqueous solution and followed by water until a neutral aqueous solution was obtained. The organic layer was dried with anhydrous $\mathrm{MgSO}_{4}$ and concentrated. $40 \mathrm{~mL}$ of toluene, $4.0 \mathrm{~g}$ $(0.039 \mathrm{~mol})$ of 1 -hexanol and $3.0 \mathrm{~g}(0.025 \mathrm{~mol})$ of so-
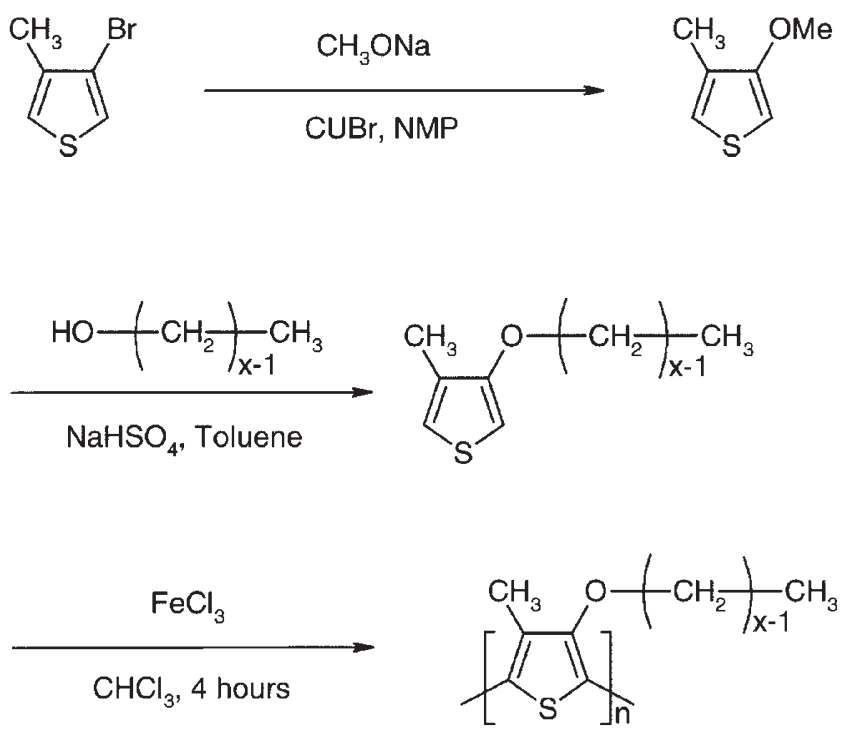

Scheme 1. Synthesis of PMThO derivatives.

dium hydrogen sulfate were added to the concentrated mixture. The mixture was refluxed for $24 \mathrm{~h}$. After cooling to room temperature, the reaction mixture was filtered and the filtrate was washed with diethyl ether. The organic layer was dried with anhydrous $\mathrm{MgSO}_{4}$ and concentrated. The mixture was purified by column chromatography. The product was obtained in a $33.4 \%$ yield $(2.58 \mathrm{~g})$.

${ }^{1} \mathrm{H}$ NMR $\left(\mathrm{CDCl}_{3}, \delta \mathrm{ppm}\right): 0.9\left(\mathrm{~m}, 3 \mathrm{H}, \mathrm{CH}_{3}\right), 1.2$ (m, 4H, $\left.\mathrm{CH}_{2}\right), 1.3\left(\mathrm{~m}, 2 \mathrm{H}, \mathrm{CH}_{2}\right), 1.7\left(\mathrm{~m}, 2 \mathrm{H}, \mathrm{CH}_{2}\right)$, $2.0\left(\mathrm{~d}, 3 \mathrm{H}, \mathrm{CH}_{3}\right), 3.9\left(\mathrm{t}, 2 \mathrm{H}, \mathrm{OCH}_{3}\right), 6.0(\mathrm{~d}, 1 \mathrm{H}$, thiophene ring), $6.7(\mathrm{~m}, 1 \mathrm{H}$, thiophene ring).

\section{3-Hexadecyloxy-4-methylthiophene (MThO16)}

3-Hexadecyloxy-4-methylthiophene was prepared in a similar manner as MThO6. The amount of reagent was $5.0 \mathrm{~g}$ of 3-bromo-4-methylthiophene, $10 \mathrm{~mL}$ of NMP, $10 \mathrm{~g}(0.041 \mathrm{~mol})$ of hexadecanol, $40 \mathrm{~mL}$ of toluene. The reaction mixture was refluxed for $27 \mathrm{~h}$. The product was obtained in a $52.0 \%$ yield $(4.98 \mathrm{~g})$. m.p. $30.2^{\circ} \mathrm{C}$.

${ }^{1} \mathrm{H}$ NMR $\left(\mathrm{CDCl}_{3}, \delta \mathrm{ppm}\right): 0.9\left(\mathrm{~m}, 3 \mathrm{H}, \mathrm{CH}_{3}\right), 1.1-$ $1.4\left(\mathrm{~m}, 26 \mathrm{H}, \mathrm{CH}_{2}\right), 1.7\left(\mathrm{~m}, 2 \mathrm{H}, \mathrm{CH}_{2}\right), 2.0(\mathrm{~d}, 3 \mathrm{H}$, $\left.\mathrm{CH}_{3}\right), 3.9\left(\mathrm{t}, 2 \mathrm{H}, \mathrm{OCH}_{3}\right), 6.1(\mathrm{~d}, 1 \mathrm{H}$, thiophene ring), $6.8(\mathrm{~m}, 1 \mathrm{H}$, thiophene ring).

\section{Poly(3-hexyloxy-4-methylthiophene) (PMThO6)}

PMThO6 was prepared by chemical oxidation method using $\mathrm{FeCl}_{3}$. One gram $(0.005 \mathrm{~mol})$ of MThO6 was added to $50 \mathrm{~mL}$ of distilled chloroform under a $\mathrm{N}_{2}$ atmosphere, and then $2.68 \mathrm{~g}(0.0165 \mathrm{~mol}, 4 \mathrm{eq})$ of $\mathrm{FeCl}_{3}$ was added to the mixture. The mixture turned black immediately. The mixture was stirred at room temperature for $4 \mathrm{~h}$. Methanol was added to the reaction mixture, and a precipitate formed. The black solid was purified by soxhlet extraction with methanol fol- 
lowing with acetone and with chloroform. The extracted polymer was purified by dissolution in small amount of chloroform and precipitation from this solution by addition of large amount of acetone. The precipitate was filtered and dried under vacuum. PMThO6 was obtained in a $5.6 \%$ yield $(56 \mathrm{mg})$.

\section{Poly(3-alkoxy-4-methylthiophene) (PMThOx)}

Other poly(3-alkoxy-4-methylthiophene)s were prepared in a similar manner as PMThO6. PMThO10; $43 \%$ yield, $\mathrm{PMThO} 12 ; 61 \%$ yield, PMThO14; 60\% yield and $\mathrm{PMThO} 20 ; 53 \%$ yield.

\section{Characterization}

${ }^{1} \mathrm{H}$ NMR measurements were carried out with a JEOL JNM-LA 400 spectrometer using $\mathrm{CDCl}_{3}$ as the solvent. DSC measurements were conducted with a Mettler DSC821 $1^{\mathrm{e}}$. Optical microscopy was performed on a Nikon polarizing optical microscope, OPTIPHOTO-POL, equipped with a Mettler FP80 controller and a FP82 hot stage. Gel permeation chromatography (GPC) was carried out with a Tosoh HLC-8020 instrument using tetrahydrofuran as the eluent, equipped with three columns (TSK gel $\mathrm{GMH}_{\mathrm{XL}}, \mathrm{GMH}_{\mathrm{XL}}$ and $\mathrm{G}^{2} 200 \mathrm{H}_{\mathrm{XL}}$ ). The instrument was calibrated with a polystyrene standard. UV-vis spectroscopy measurements were carried out with a HITACHI U-3410 spectrophotometer equipped with a Mettler FP80 controller and a FP82 hot stage. X-Ray diffraction patterns were recorded with a RIGAKU RINT2500 with Ni-filtered $\mathrm{Cu} \mathrm{K} \alpha$ radiation. The sample in quartz capillary (diameter $1 \mathrm{~mm}$ ) was held in a temperature-controlled cell (RIGAKU LC high temperature controller). The electric conductivity was measured with a Mitsubishi Chemical Loresta HP (MCP-T410) electrometer by using four-probe (Mitsubishi Chemical MCP-TP06P) technique at room temperature. The size of cast films on the glass substrate and free-standing films of polythiophene derivatives is about $20 \mathrm{~mm} \times 15 \mathrm{~mm}$. The thickness of the cast films and the free-standing films is about $10-30 \mu \mathrm{m}$. Immediately after the cast film on the glass substrate and the free-standing films were doped with iodine vapor for $24 \mathrm{~h}$, the electric conductivity of the films was measured by four-probe technique at room temperature in air. The doped freestanding was placed on the glass substrate and then the electrical conductivity of the doped films was measured.

\section{RESULTS AND DISCUSSION}

Figure 1 showed the chemical structure of synthesized PMThO derivatives. We prepared PMThO derivatives to investigate the effect of the length of the side chain on the physical properties such as optical

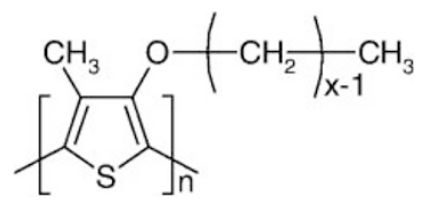

PMThOx $(x=6,10,12,14,16,20)$

Figure 1. Chemical structure of polythiophene derivatives.

Table I. Molecular weights and polydispersity of PMThO derivatives

\begin{tabular}{lcccr}
\hline Polymers & $\bar{M}_{\mathrm{n}}$ & $\bar{M}_{\mathrm{w}}$ & $\bar{M}_{\mathrm{w}} / \bar{M}_{\mathrm{n}}$ & $\mathrm{DP}^{\mathrm{a}}$ \\
\hline PMThO6 & 18000 & 36000 & 2.0 & 91 \\
PMThO10 & 32000 & 53000 & 1.7 & 126 \\
PMThO12 & 49000 & 81000 & 1.7 & 173 \\
PMThO14 & 39000 & 65000 & 1.7 & 126 \\
PMThO16 & 38000 & 66000 & 1.7 & 112 \\
PMThO20 & 60000 & 100000 & 1.7 & 152 \\
\hline
\end{tabular}

${ }^{\text {a }}$ Degree of polymerization

and electrical properties.

The PMThO derivatives obtained are dissolved in organic solvents such as chloroform and tetrahydrofuran (THF). Molecular weights of PMThO derivatives were determined using GPC. The number-average molecular weight, polydispersity and degree of polymerization of PMThO derivatives are summarized in Table I. Molecular weights of PMThO derivatives are relatively large and the polydispersity is not so large.

It is possible to estimate the regioregularity of PMThO derivatives using ${ }^{1} \mathrm{H}$ NMR and ${ }^{13} \mathrm{C}$ NMR measurements. A typical ${ }^{1} \mathrm{H}$ NMR spectrum of PMThO derivatives is shown in Figure 2a. Figure $2 b$ displays the expanded ${ }^{1} \mathrm{H}$ NMR spectrum in the range of $2.0-2.6 \mathrm{ppm}$. Several peaks are observed in the range of $2.0-2.3 \mathrm{ppm}$ in the ${ }^{1} \mathrm{H}$ NMR spectrum. The

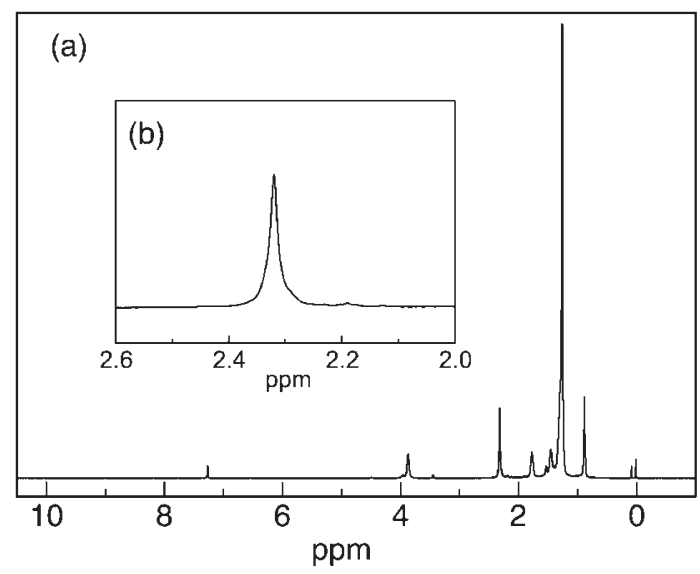

Figure 2. ${ }^{1} \mathrm{H}$ NMR spectrum (a) including expanded spectrum in the range of 2.0-2.6 ppm (b) of PMThO14 in $\mathrm{CDCl}_{3} ; \delta$ (ppm) relative to TMS. 
Table II. Regioregularity of PMThO derivatives

\begin{tabular}{lc}
\hline Polymers & $\begin{array}{c}\text { Regioregularity }^{\mathrm{a}} \\
(\%)\end{array}$ \\
\hline PMThO6 & 88 \\
PMThO10 & 91 \\
PMThO12 & 96 \\
PMThO14 & 96 \\
PMThO16 & 95 \\
PMThO20 & 94 \\
\hline
\end{tabular}

adetermined by ${ }^{1} \mathrm{H}$ NMR spectroscopy measurements

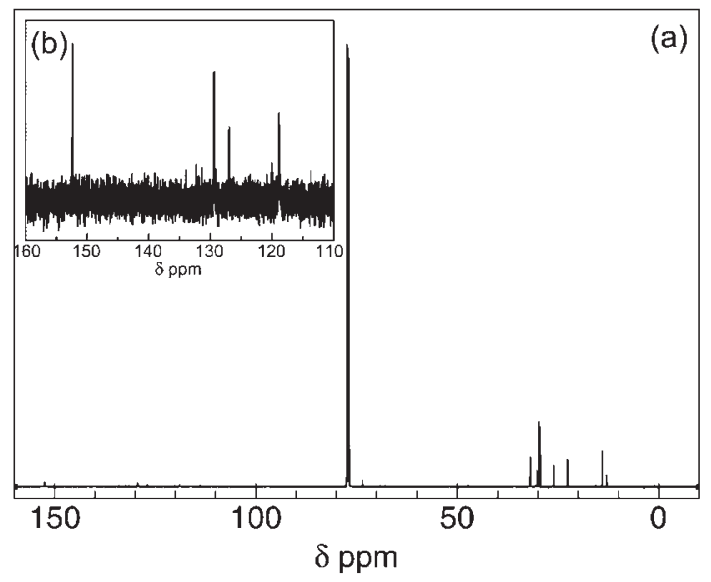

Figure 3. ${ }^{13} \mathrm{C}$ NMR spectrum (a) including expanded spectrum in the range of 110.0-160.0 ppm (b) of PMThO14 in $\mathrm{CDCl}_{3}$; $\delta(\mathrm{ppm})$ relative to TMS.

large peak at $2.32 \mathrm{ppm}$ assigned to the methyl group at the 4-position in the thiophene ring displays HT-HT coupling. ${ }^{9}$ Other small peaks are assigned to the regioisomers. The content of HT-HT coupling in the polythiophene backbone is estimated by the integration of the peaks. The content of HT-HT coupling (regioregularity) in the polymer backbone is summarized in Table II. The regioregularity of PMThO derivatives with relatively longer side chain is above $90 \%$. PMThO12 and PMThO14 display the highest regioregularity. This result indicates that dodecyloxy or tetradecyloxy side chain would be proper to obtain PMThO derivatives with higher regioregularity in the polymer backbone.

Regioregularity of PMThO derivatives can be also confirmed by ${ }^{13} \mathrm{C}$ NMR measurements. Figure $3 \mathrm{a}$ displays a typical ${ }^{13} \mathrm{C}$ NMR spectrum of PMThO derivatives. Figure $3 b$ shows the expanded aromatic region in ${ }^{13} \mathrm{C}$ NMR spectrum of PMThO derivatives. The four sets of ${ }^{13} \mathrm{C}$ resonances can be detected in the aromatic region between 115 and $155 \mathrm{ppm}$. The four sets of ${ }^{13} \mathrm{C}$ resonances correspond to four carbons in the thiophene ring. If PMThO derivatives were a regiorandom structure in the polymer backbone, 16 sets of ${ }^{13} \mathrm{C}$ resonances are detected. ${ }^{9,12,18,19}$ The ${ }^{13} \mathrm{C}$ NMR spectrum of PMThO derivatives supports the interpre-

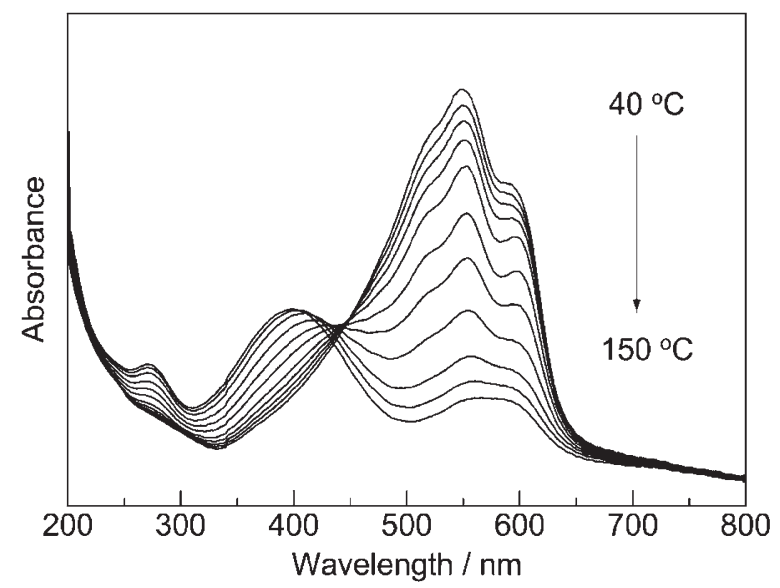

Figure 4. Temperature dependence of UV-vis spectra of PMThO14 in the film state.

tation of the ${ }^{1} \mathrm{H}$ NMR data. ${ }^{1} \mathrm{H}$ NMR and ${ }^{13} \mathrm{C}$ NMR data of PMThO derivatives demonstrate that PMThO derivatives with regioregular structure in the polymer backbone can be easily obtained by the conventional oxidative polymerization using iron trichloride as a catalyst.

Temperature dependence of UV-vis spectrum for PMThO derivatives in the film state is shown in Figure 4. Vibronic peaks characteristic of high regioregular structure in the polymer backbone are observed around $540 \mathrm{~nm}$ in the UV-vis spectrum. The intensity of the peaks decreases with increasing temperature. New peaks appear near $275 \mathrm{~nm}$ and $400 \mathrm{~nm}$ in the UV-vis spectrum of PMThO derivatives with increasing temperature. The color of PMThO derivatives in the film state changes from violet to dark yellow with increasing temperature. The temperature dependence of UV-vis spectrum displays that PMThO derivatives have co-planar structure of the polymer backbone at low temperature, while at high temperature, they have non-planar structure of the polymer backbone. ${ }^{16,17}$ The reversible temperature dependence of UV-vis spectrum of PMThO derivatives is detected. This result demonstrates that the conformational change of the polymer backbone is also reversible. Absorption maxima of UV-vis spectra and band gap energy of PMThO derivatives are summarized in Table III. The band gap energy of PMThO derivatives was obtained by UV-vis spectrum of PMThO derivatives. The band gap energy of PMThO derivatives is about $1.9 \mathrm{eV}$ that is lower than that of conventional polythiophenes. $^{8}$

Figure 5 shows the X-Ray diffraction pattern of PMThO6 at room temperature. A sharp peak at $4.9^{\circ}$ and a broad peak in the wide angle region are observed. The sharp peak in the small angle region relates to the lamellar stacking formed by polythiophene backbones of PMThO derivatives. ${ }^{22,23}$ The $d$-spacing 
Table III. Absorption maxima and band gap energy of PMThO derivatives

\begin{tabular}{lcc}
\hline Polymers & $\begin{array}{c}\text { Absorption maxima } \\
(\mathrm{nm})\end{array}$ & $\begin{array}{c}\text { Band gap energy } \\
(\mathrm{ev})\end{array}$ \\
\hline PMThO6 & 549 & 1.92 \\
PMThO10 & 547 & 1.93 \\
PMThO12 & 542 & 1.94 \\
PMThO14 & 541 & 1.91 \\
PMThO16 & 546 & 1.92 \\
PMThO20 & 550 & 1.92 \\
\hline
\end{tabular}

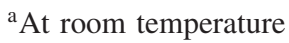

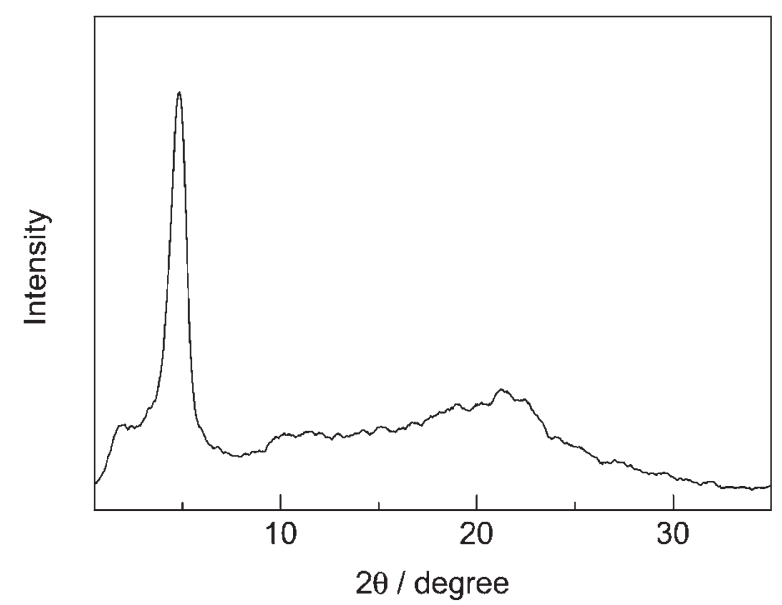

Figure 5. X-Ray diffraction pattern of PMThO6 at room temperature.

based upon the sharp peak in the small angle region is $18.2 \AA$. On the other hand, the peak in the wide angle region $\left(2 \theta=\right.$ about $\left.22^{\circ}\right)$ is assigned to the intermolecular $\pi$ stacking between thiophene rings. ${ }^{22,23} \mathrm{~A}$ shoulder peak near $2^{\circ}$ and a broad peak near $10^{\circ}$ are also detected in the X-Ray diffraction pattern of PMThO6. However, the origin of the peaks is not clarified at the present time. Figure 6a also displays the X-Ray diffraction pattern of PMThO14. The XRay diffraction pattern of PMThO14 is similar to that of PMThO6. The $d$-spacing based upon the sharp peak in the small angle region is $30.0 \AA$. The intensity of the sharp peak of PMThO14 is slightly stronger than that of PMThO6. It is identified by comparing the intensity of the sharp peak in the small angle region with that of the broad peak in the wide angle region. This result indicates that the polythiophene backbones of PMThO derivatives with long alkoxy chains would form the lamellar stacking tightly compared to that of PMThO derivatives with short alkoxy chains. The $d$-spacing based upon the sharp peak in the small angle region increases with increasing length of the side chain of PMThO derivatives as shown in Figure $6 \mathrm{~b}$.

The electrical conductivity of PMThO derivatives in both free-standing and cast films were investigated. The free-standing films of PMThO derivatives can be

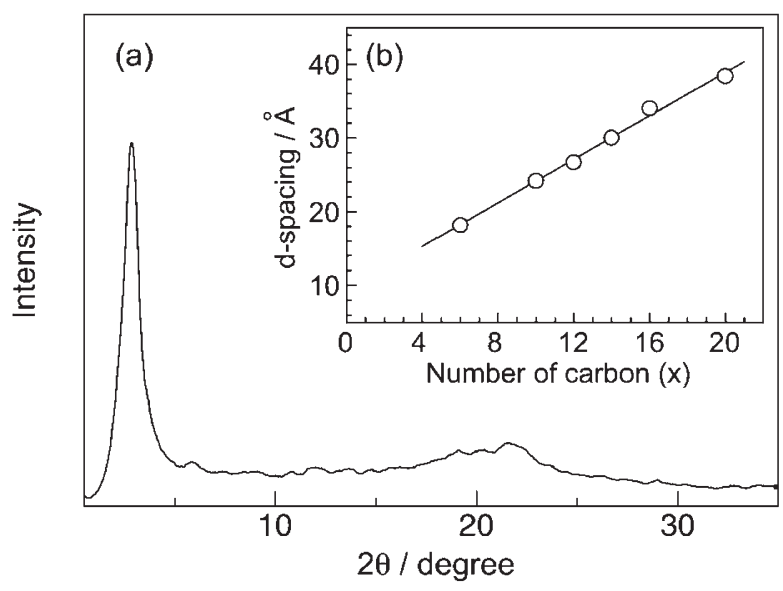

Figure 6. X-Ray diffraction pattern of PMThO14 at room temperature (a) and the $d$-spacing based upon the peak in the small angle region of the X-Ray diffraction pattern for PMThO derivatives (b).

Table IV. Electrical conductivity of PMThO derivatives

\begin{tabular}{lcc}
\hline Polymers & $\begin{array}{c}\text { Cast film } \\
\left(\mathrm{S} \mathrm{cm}^{-1}\right)\end{array}$ & $\begin{array}{c}\text { Free standing film } \\
\left(\mathrm{S} \mathrm{cm}^{\mathrm{a}}\right)\end{array}$ \\
\hline PMThO6 & 0.05 & 0.04 \\
PMThO10 & 1.44 & 2.74 \\
PMThO12 & 1.07 & 3.76 \\
PMThO14 & 1.44 & 5.25 \\
PMThO16 & 1.20 & 3.00 \\
PMThO20 & 1.07 & 1.78 \\
\hline
\end{tabular}

${ }^{\mathrm{a}}$ After doping with $\mathrm{I}_{2}$ vapor for $24 \mathrm{~h}$

easily obtained from the cast film. The free-standing films and cast films on the glass substrate were doped with iodine vapor for $24 \mathrm{~h}$. The electrical conductivity of PMThO derivatives were measured by fourprobe technique at room temperature in air. The conductivity of PMThO derivatives are summarized in Table IV.

The regioregularity of PMThO derivatives with relatively longer side chain (PMThO12, PMThO14, PMThO16 and PMThO20) was almost same value. The electrical conductivity of the PMThO derivatives was about $10^{0} \mathrm{~S} \mathrm{~cm}^{-1}$ in spite of cast and free standing films. The electrical conductivity of PMThO10 was also about $10^{0} \mathrm{~S} \mathrm{~cm}^{-1}$, although the regioregularity of PMThO10 was lower than that of the PMThO derivatives with relatively longer alkoxy chain. Both the regioregularity and the electrical conductivity of PMThO6 were lower compared to those of other PMThO derivatives. The lower value of the electrical conductivity of PMThO6 would originate from smaller molecular weight, lower regioregularity and looser lamellar stacking formed by the polythiophene backbone.

The electrical conductivity of PMThO derivatives was lower than that of poly(3-hexylthiophene).${ }^{9}$ A dif- 
ference of the electrical conductivity between PMThO derivatives and poly(3-hexylthiophene) would arise from the difference of the chemical structure of the monomer unit, the difference of the regioregularity or the difference of the doping level. However, details of the reason why the electrical conductivity of PMThO derivatives was lower than that of poly(3-hexylthiophene) are not clarified at the present time.

\section{REFERENCES}

1. A. Pron and P. Rannou, Prog. Polym. Sci., 27, 135 (2002).

2. K. Yoshino, S. Nakayama, and R. Sugimoto, Jpn. J. Appl. Phys., 26, L1038 (1987).

3. M. Sato, S. Tanaka, and K. Kaeriyama, Makromol. Chem., 188, 1763 (1987).

4. S. Hotta, S. D. D. V. Rughooputh, A. J. Heeger, and F. Wudl, Macromolecules, 20, 212 (1987).

5. M. Leclerc, F. M. Diazu, and G. Wegner, Makromol. Chem., 190, 3105 (1989).

6. M. Sato and H. Mori, Polym. Commun., 32, 42 (1991).

7. G. Zerbi, B. Chierichetti, and O. Inganas, J. Chem. Phys., 94, 4646 (1991).

8. H. S. O. Chan and S. C. Ng, Prog. Polym. Sci., 23, 1167 (1998).

9. T.-A. Chen, X. Wu, and R. D. Rieke, J. Am. Chem. Soc.,
117, 233 (1995).

10. X. Wu, T.-A. Chen, and D. Rieke, Macromolecules, 29, 7671 (1996).

11. R. D. McCullough and R. D. Lowe, J. Chem. Soc., Chem. Commun., 1992, 70.

12. R. D. McCullough, R. D. Lowe, M. Jayaraman, and D. L. Anderson, J. Org. Chem., 58, 904 (1993).

13. R. D. McCullough and S. P. Williams, J. Am. Chem. Soc., 115, 11608 (1993).

14. M. Leclerc, M. Fréchette, J.-Y. Bergeron, M. Ranger, I. Lévesque, and K. Faïd, Macromol. Chem. Phys., 197, 2077 (1996).

15. I. Lévesque and M. Leclerc, Macromolecules, 30, 4347 (1997).

16. C. Roux, J.-Y. Bergeron, and M. Leclerc, Makromol. Chem., 194, 869 (1993).

17. M. Leclerc, C. Roux, and J.-Y. Bergeron, Synth. Met., 55-57, 287 (1993).

18. K. Faïd, M. Fréchette, M. Ranger, L. Mazerolle, I. Lévesque, and M. Leclerc, Chem. Mater., 7, 1390 (1995).

19. I. Lévesque and M. Leclerc, Chem. Mater., 8, 2843 (1996).

20. K. Tanaka, T. Mihara, and N. Koide, unpublished data.

21. L. Groenendaal, G. Zotti, and F. Jonas, Synth. Met., 118, 105 (2001).

22. R. D. McCullough, S. Tristram-Nagle, S. P. Williams, R. D. Lowe, and M. Jayaraman, J. Am. Chem. Soc., 115, 4910 (1993).

23. S.-A. Chen and J.-M. Ni, Macromolecules, 25, 6081 (1992). 